\title{
Research Article \\ Research on Human Resource Recommendation Algorithm Based on Machine Learning
}

\author{
Hong Zhu $\mathbb{i D}^{1,2}$ \\ ${ }^{1} X i$ 'an Jiaotong University, Xi'an, Shaanxi 710049, China \\ ${ }^{2}$ Shaanxi XueQian Normal University, Xi'an, Shaanxi 710100, China \\ Correspondence should be addressed to Hong Zhu; 11009@snsy.edu.cn
}

Received 21 June 2021; Revised 2 August 2021; Accepted 13 August 2021; Published 25 August 2021

Academic Editor: Muhammad Usman

Copyright (๑) 2021 Hong Zhu. This is an open access article distributed under the Creative Commons Attribution License, which permits unrestricted use, distribution, and reproduction in any medium, provided the original work is properly cited.

\begin{abstract}
The economic environment has changed dramatically around the world in recent years, generating favorable conditions for the growth of small- and medium-sized firms. The socioeconomic development and international integration of China are greatly influenced by the growth in both quality and quantity, the scale of operations, and the internal force of small- and medium-sized businesses. Moreover, in comparison with other developed countries around the world, Chinese small- and medium-sized enterprises continue to face many limitations in terms of size and contribution levels and have not yet fully realized their potentials due to difficulties and poor quality; human resources in this field are still lacking. This study defines the current state of human resources in small and medium firms, the factors that impede development, and the steps that can be taken to overcome these obstacles in order to assist human resource development in this sector during the current period. This study uses machine learning (ML) techniques to manage and analyze human resource data in modern enterprises. The ML techniques realize the functions of the human resource system and reduce the business volume in human resource in order to improve the efficiency and management of the human resource work. In this paper, we designed and implemented the wage forecasting model in human resources that uses a gradient descent algorithm, its types, and backpropagation (BP) neural network to improve the accuracy of the forecasting model. We performed multiple experiments by using a various number of neurons in the hidden layers, different number of iterations, and several types of gradient descent algorithms. The BP neural network model was performed brilliantly by attaining the training accuracy of $89.98 \%$ and validation accuracy of $84.05 \%$. The experimental results show the significance and importance of the proposed work.
\end{abstract}

\section{Introduction}

Human resource management system (HRMS) is an important tool of enterprise management that plays an important role in the development of enterprises. From its birth to now, HRMS has experienced three stages [1]. The first generation of HRMS appeared in the 1960s. The main objective and functionality of HRMS at that time was to automatically calculate the salaries of employees via a computer system, excluding salary data analysis and nonfinancial information. The main reason for such circumstances at that time was the limited resources and technical requirements. The second generation of HRMS started in the early 1980s. The main drawbacks of the first generation were overcome in the second generation with the development of the database technology and other requirements missing in the first generation. The functions of nonfinancial information, salary data analysis, and report production were kept in consideration, which greatly improved the practicability of HRMS in the enterprises. However, there were still some major problems in the second generation of HRMS; for example, the management process was not standardized and the information processing techniques were not comprehensive. The third generation of HRMS appeared in the late 1990s. Because of the fierce competition among enterprises at that time, especially in the introduction and cultivation of talent, HRM has risen to the top of the company's priority list. Due to the popularity of computers at that time, different technologies, particularly database technology and Internet, had made great progress in the 
HRMS. These technologies consolidate human resources for unified management and create integrated data sources, making data analysis and sharing easier.

With the advent of the information age and the development of enterprises, a vast amount of data is produced which needs to be managed and controlled in a well-organized manner. To control, manage, and analyze such a large amount of data is indeed a tough task for human beings to implement at all, so the existence of information management system (MIS) came into being. Management information system (MIS) is management software having the functionality of data collection, database creation, and data management (including data storage and extraction) and can analyze a large amount of data [2-4]. The HRMS is an information management system which combines both HRM and information technology (IT). It is not only an information processing tool but also a resource management standard. Its main purpose is to standardize the business process of the human resource department through the system, concentrate human resource information, and improve the transparency of HRM. HRMS plays an important role in enterprises and has the powerful functions of optimizing business processes, improving work efficiency, and improving management mode. Its quality is directly related to the performance of the enterprise, which affects the survival and development of the enterprise $[5,6]$. Therefore, the HRMS is considered an important tool for enterprises. However, in recent years, the data collected by enterprises are growing rapidly and continuously. Such a large amount of data has exceeded the processing capacity of HRMS, which leads to its failure to conduct data management and data analysis normally. This situation is described as "rich data, but the information is poor." Furthermore, the majority of the enterprise-level HRMS has not been fully utilized, with only data collection and storage, no effective data management and analysis, and no use of a large amount of data, wasting not only the enterprise's limited resources but also the opportunity to use big data to develop rapidly. Due to the fact that the traditional HRMS cannot fulfill the needs of organizations' while having big data, in order to make the system work normally, it must artificially screen and process such a vast amount of data, thereby reducing the volume of big data. However, this kind of processing method is back to the old era of manual data processing, which seriously affects the improvement and efficiency of the work. Therefore, people started the use of data mining techniques to reduce the data to be processed $[7,8]$. The purpose of data mining is to analyze and find out the important data patterns in the massive amount of raw data and finally convert the original data into useful information and knowledge.

The 21st century is the era of big data that brought a revolution in every aspect of human life such as healthcare, industries, government sectors, social media, and business organization and changed the policies of human resource management $[9,10]$. The traditional HRMS can realize the functionality of data entry, query, and statistics but cannot analyze the relationship between data samples effectively and cannot predict the future development of enterprises according to the existing data. To solve this problem, people put forward using data mining techniques to extract and analyze big data. Machine learning (ML) is one of the main tools to realize data mining, and it has various applications in different fields, including the field of HRM and businesses. The important roles of ML in human resource management include recruitment, talent management, preventing brain drain, and improving human resource precise management and are analyzed in detail by different researchers $[11,12]$. In a study conducted by Chen et al. [13], they presented the capability of data mining techniques in the development of the decision-making process of HRMS. The significance of data mining in turnover management is analyzed and implemented by Meng [14]. They presented a knowledge discovery approach to assist firms in predicting the future performance of their employees and then assigning relevant personnel to the appropriate project positions.

In this paper, a fuzzy clustering method based on an adaptive genetic algorithm is proposed that provides effective data analysis for the performance appraisal of employees in human resource management of modern enterprises. The potential of AI in HRM is explored in much detail in different studies, among which six scenarios are turnover prediction of the artificial neural network (ANN), candidate search based on a knowledge search engine, personnel scheduling of genetic algorithm, HR emotion analysis, resume data collection and extraction of information, and interactive voice response employee self-service. Some of the basic contributions of this paper are given as follows:

(1) This study designs and implements the model of human position matching using ML algorithms, which can provide a guarantee for effective human resource allocation.

(2) A new method is proposed to evaluate the candidates in an online recruitment system and to solve the problem of candidate ranking using ML algorithms. Further, a new algorithm based on support vector regression is designed for the prediction of the human resource demand of enterprises which has a good practical reference value.

(3) To solve the problem of staff turnover, an extreme gradient boosting (XGBoost) algorithm is used to predict the employee turnover rate. It is proved with the help of experimental results that it is more accurate than the other supervised classification algorithms in predicting employees' turnover.

(4) Based on the work and life situation, this study analyzes the factors affecting the retirement of employees and uses ML algorithms to create a retirement prediction model, which can provide solutions for the outflow of human resources. Further, this study realizes a human resource ranking model, which can predict the ranking and classification of resumes with high accuracy, and effectively simplifies the work of human resources.

The rest of the paper is organized as follows. Section 2 represents the related work, while Section 3 illustrates the applications of the backpropagation neural network (BPNN) 
in wage forecast. Section 4 demonstrates the experimental simulations and results analysis, and finally, Section 5 concludes the research work.

\section{Related Work}

The term "human resources" was initially coined in the early 1970s, when the importance of labor relations became more widely recognized, and concepts like motivation, organizational behavior, and selection assessments began to emerge. In "The Human Resources Glossary," Huong [15] describes human resource management as "the people and staff who administer an organization," as opposed to an organization's financial and material resources. ML is a subject that studies how to improve the performance of the system itself using calculation and experience. In general, it transforms the unordered data into useful information through calculation, and this information generally refers to the model obtained through training and learning. With the use of computer systems for human resource tasks, HRM has experienced significant changes since the 1980s. Initially, human resource professionals used these systems primarily as record-keeping systems for simple operations like accepting and storing job applications on behalf of their employers [16]. However, as information technology (IT) advanced, HR professionals began to employ sophisticated computer systems to do the majority of their everyday tasks and make strategic decisions. As a result, HR procedures have been greatly simplified [17]. IT is increasingly and widely used in HRM, and complex computer systems are required for both internal and external operations such as recruitment, performance assessment, remuneration, outsourcing, compensation, onboarding, development, strategic analytics, and business-to-employees (B2E). The advancement in computer engineering has resulted in the emergence of advanced technologies like artificial intelligence (AI). AI is also bringing up significant changes. HRM, a tech-dependent phenomenon, has recently seen such a shift. These significant shifts in HRM that we are witnessing are well worth investigating. The industrial revolution in the 18th century caused a significant shift in industries and economics [18]. These significant shifts in HRM that we are witnessing are well worth investigating [19]. Manufacturers encountered the scientific management paradigm in the early 20th century, which promoted standard-performance production as the optimum approach to achieve maximum profitability [20]. Employers at the time were more concerned with employee production rather than with employee satisfaction, owing to the application of scientific management techniques and the lack of governmental regulation and law governing personnel policies and practices. The widespread study of human behavior, as well as dramatic changes in the structure and intensity of worldwide competition between large organizations, had a significant impact on personnel management methods near the end of the 20th century, and as a result, HRM emerged [21].

HRM has become the standard technique for managing people inside a business since the early 1980s [22]. It has been highlighted as an important method for developing management systems that effectively steer human competencies toward corporate goals since then [23]. With the rise of the Internet as a professional platform and technological advancements, human resource information is evolved into a central hub for human resource management. Electronic $\mathrm{HR}$ is the more generic term for the use of computer technology in HR [24]. E-HRM appears to have become the prevalent word for referring to virtual HR or e-enabled HRM in the last two decades, in which IT is used to implement comprehensive HR functions within a business $[25,26]$. In general, it is regarded to be useful since it is designed to improve HR efficiency, save costs, minimize administrative responsibilities, simplify HR planning, and empower HR practitioners to become strategic or business partners in firms. According to Marler and Parry [27], e-HRM may not be as cost-effective as it claims to be. Organizations have been transitioning from traditional faceto-face HR procedures to current electronic ones; e-HRM is becoming an important theoretical and practical phenomenon $[28,29]$. Many individuals expected e-HRM to revolutionize the way HRM is done in organizations, moving it away from being purely administrative and toward being more strategic. According to Thite et al. [30], the electronic transition faces two major hurdles in the perspectives of business and technology leaders. One is HR's ability to assist business leaders in adopting a digital mindset, as well as a digital way of managing, organizing, and leading change. The second is HR's ability to transform the whole experience of employee by transforming HR processes, systems, and the HR organization via novel digital platforms, applications, and way to deliver HR services.

Information technology (IT) is on many people's agendas, especially professionals and academics, as a crucial commercial tool for many firms. What is more intriguing is that there's a global effort underway to improve IT's qualities using AI, often known as cognitive computing. According to Pan [31], since 2010, several IT behemoths have bought approximately 140 AI start-ups. These businessmen are attempting to combine artificial intelligence (AI) into a variety of computer systems in order to produce seamless experiences during the installation of these technologies. AI strives to make machines capable of performing tasks in the same way that humans do. However, given the fact that people have not yet completely deciphered and grasped the human intelligence that AI simulates, Shah and Warwick [32] believe that computers functioning like human beings should be viewed in terms of imitation. The Japanese Cabinet has announced a new project called Society 5.0 with the goal of bringing about societal improvement. This new approach appears to be aimed at achieving a high level of human-machine interaction [33]. Its goal is to connect people, objects, and computers in cyberspace, using sensors to detect commands and AI to execute them [34]. Unlike the idea of Business 4.0, which we are approaching, Society 5.0 is not limited to the industrial industry but rather penetrates social life as AI, the Internet of Things (IoT), augmented reality, and robotics come together [35].

In this paper, a new HR recommendation algorithm based on the hidden semantic model and the deep forest is 
proposed in document, which can better recommend the interesting posts for the users. Various studies made an attempt to understand that whether machine learning (ML) can be effectively used to perform potential assessments using preventive methods, thus helping or even replacing human resource managers. The ML methods are used in the earlier studies to provide human resource decision support. In this paper, a model of employee turnover based on a gradient descent algorithm and BP neural network is established in document, which can predict the turnover of employees in enterprises and provide enterprises with the opportunity to solve any problems and improve the retention rate. To solve the problem of human resource replacement in technical posts, this study uses supervised ML techniques to predict the appropriate human resources to fill the vacancy of the technical posts. All of these indicate that the influence of ML in the field of human resource management is expanding and has a great potential to improve the efficiency and performance of HRMS.

\section{Applications of ML and Backpropagation Neural Network (BPNN) in Wage Forecast}

According to R. Michalski, research in the field of ML can be divided into "learning from examples," "learning in problem-solving and planning," "learning through observation and discovery," and "learning from instructions." According to E. A. Feigenbaum, in the manual of AI, ML can be divided into "mechanical learning," "teaching-learning," "analogy learning," and "inductive learning." At present, inductive learning is the most widely used learning technique of ML. There are three main streams of inductive learning: connectionist learning based on neural networks, semiotic learning, and statistician learning. Connectionism learning based on neural networks is learning of the ML model that simulates the functionality of the neurons of the human brain, and its work in terms of ML is represented by the perceptron, although connectionism learning started early and encountered great obstacles. The main reason was that the neural network at that time could only deal with a simple linear problem and even could not solve the small problem of XOR, which led to the development and learning of the neural networks. Semiotic learning was originated from mathematical logic, and its core idea is to predict the results through the deduction and inverse deduction of symbols, which mainly represents decision tree and logic-based learning. Statistical learning is used to apply statistical learning knowledge to ML techniques, and its representative technology is SVM and "kernel method."

Neural network is a parallel interconnected network composed of adaptive simple units, and its organization can simulate the interaction of biological neural system to realworld objects. The "simple unit" in the definition of a neural network refers to the neuron model, and the neuron model generally refers to the "MP neuron model or single-layer perceptron model" proposed by McCulloch and others in 1943 [14]. In the model, firstly, the neurons get input signals from the environment and then multiply them with the corresponding weights put on the edges. The output of a neuron then becomes the input for another neuron and this process continues until it reached the last layer. Therefore, neurons can calculate the total input signal by multiplying itself with the corresponding weights, then compare it with the threshold value of neurons, and process it via the activation function. At last, the output of the neuron is obtained, as shown in Figure 1.

A neural network is a topological structure consists of various neurons connected via a particular hierarchical structure. Neural network models can be classified into two types based on the hierarchical structure of neurons: interconnection network model and hierarchical network model. The neurons in the hierarchical network model are hierarchical and are generally divided into several layers according to their functions, and each layer is connected, such as the input layer, the middle layer, and the output layer. The interconnection network model is that in which any two neurons can be connected and has certain randomness. The hierarchical network has become the most widely used model structure because of its good structure and easy analysis.

As mentioned earlier, the learning process of the neural network is to adjust the parameters' values of neurons, and the backpropagation (BP) algorithm is used to achieve this purpose in order to reach the target. BP algorithm is based on gradient descent strategy, which takes the negative gradient direction of the target as the search direction to adjust the parameters and finally achieves convergence by continuously iterating and updating the parameters. It is one of the most successful neural network algorithms and has been used widely in various applications. In general, the BPNN model refers to the multilayer feed-forward neural network trained by the BP algorithm, as shown in Figure 2.

3.1. Model Selection. Salary prediction is a kind of regression problem. The regression prediction models commonly used in ML include linear regression, polynomial regression, ridge regression, lasso regression, elastic network regression, and neural networks. Linear regression is a regression model composed of linear variables. Therefore, the linear relationship between the independent variables and dependent variable must be satisfied; that is, linear regression is only applicable to solve linearly separable problems. In linear regression problems, there exists a linear relationship between variables; as a result, the calculation complexity is low and the training speed is faster. The disadvantage of linear regression is that it is very sensitive to outliers. If there is any outlier in the data, the accuracy of the model will be affected. Polynomial regression is the extension of linear regression, which refers to the regression model with independent variables, having an index greater than 1 in the regression equation. When the independent index is equal to 1 , the polynomial regression equation degenerates and becomes a linear regression equation. As in most of the cases, independent variables and dependent variables are not linear, so the polynomial regression is an automatic choice for it, which can solve nonlinear and complex problems with ease. The disadvantage of the polynomial regression model is that 


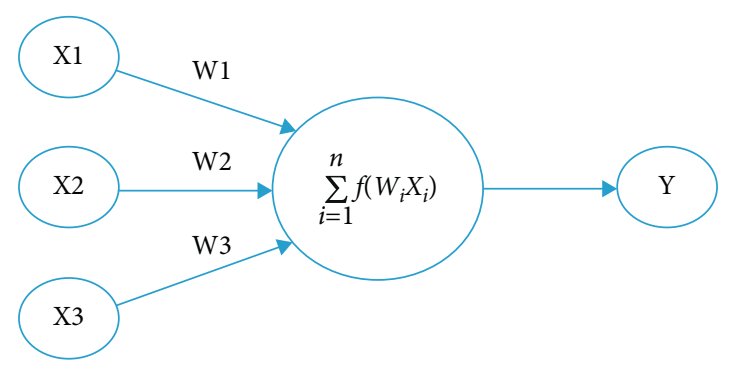

Figure 1: Single-layer perceptron model.

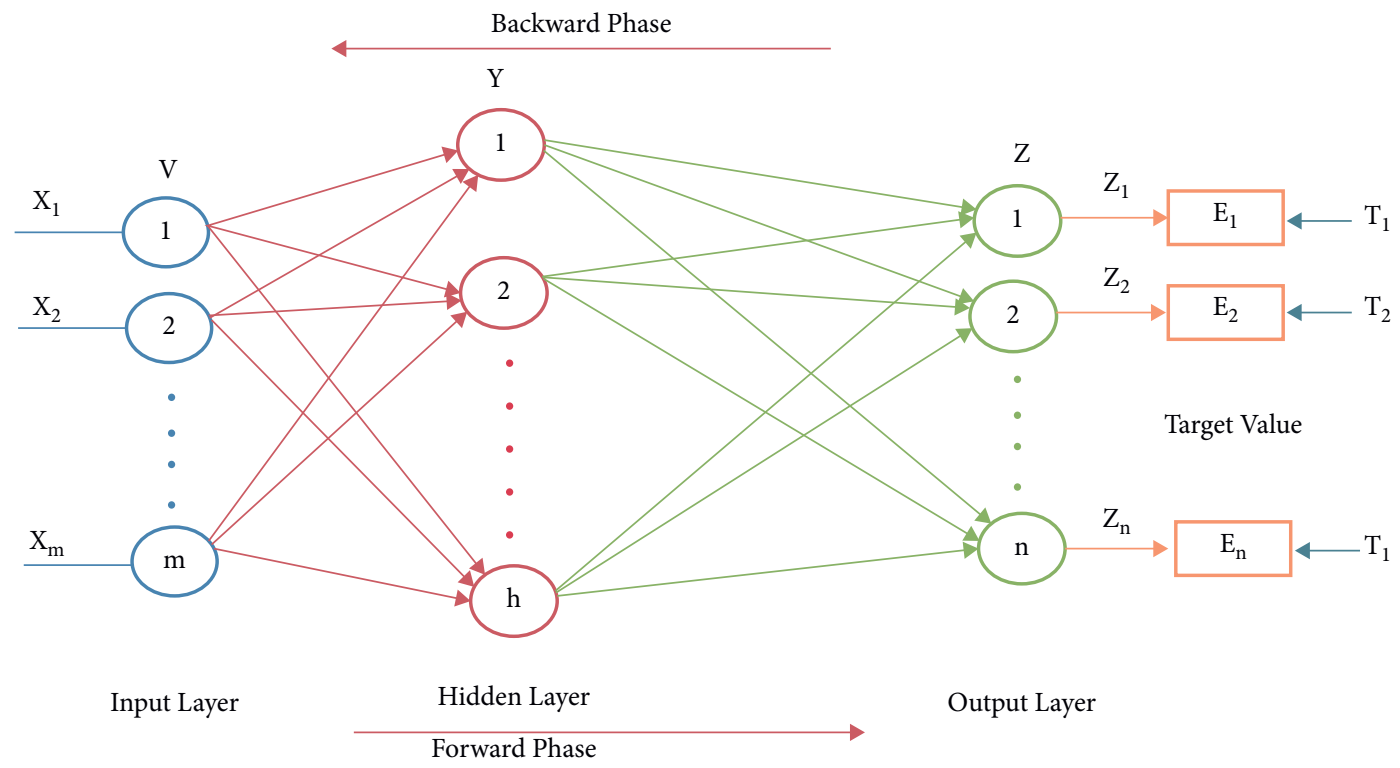

FIGURE 2: General architecture of backpropagation neural network (BPNN) model.

the best index is difficult to determine, the high index is easy to cause overfitting, and the low index is prone to underfitting.

Ridge regression is an optimization technique used when the data features of linear regression or polynomial regression have multiple collinearity (i.e., there is an approximately linear correlation between independent variables). It reduces the influence of multiple collinearities by adding a square bias factor to the regression estimation function (loss function). Lasso regression is similar to ridge regression, and it is also an optimization technique used when the data features of linear regression or polynomial regression have multiple collinearities and also add bias factors to regression estimation function. Only the square offset is added in ridge regression, and the absolute value offset is added in the lasso regression. Elastic net regression is a mixture of ridge regression and lasso regression. It adds both square bias and absolute value offset in regression estimation function, to achieve the common effect of ridge regression and lasso regression. BP neural network is the most widely used type of neural network. Theoretically, a three-layer BP neural network can approach any continuous function with arbitrary precision, has a strong learning ability and simple network structure, and takes low time in calculation. At the same time, neural networks have some shortcomings too. The most important and commonly faced problem in neural networks is the topological structure, parameters initialization, and optimization techniques of neural network. Further, it has a great impact on the training results of the model; if the selection of superparameters is not good, it will significantly affect the performance of the model.

3.2. Salary Forecast Model Based on BP Neural Network. The BP neural network offers nonlinear mapping, generalization, and fault tolerance capabilities. Moreover, the multilayer (three-layer or above) BP neural network can approximate any precision nonlinear function theoretically, which is a powerful ML algorithm. To establish a salary forecast model based on BP neural network, it is necessary to solve the key problems such as topology, activation function selection, and parameter initialization.

The design of the network topology is relatively free and has no fixed form. The hierarchical structure is generally designed according to the nature of the problem. In most cases, the network parameters are also selected according to the nature of the problem and earlier experience. Salary prediction is a mapping of multiple input and a single output. According to the format of data in demand analysis, it can be determined that the number of input layer neurons 
in the network is 14 and the number of neurons in the output layer is 1 . There is no definite calculation method for the number of neurons in the hidden layers. Some methods can be used to estimate its approximate range, and then the model can be trained repeatedly using the trial and error method. Finally, the number of neurons in a hidden layer can be confirmed according to the performance of the model. There are many empirical formulas used to calculate the number of neurons in the hidden layer. The most commonly used formulas to calculate the number of neurons in the hidden layer are given as follows:

$$
\begin{aligned}
& n_{h}=\sqrt{n_{i} \cdot n_{o}}, \\
& n_{h}=\sqrt{n_{i} \cdot n_{o}}+k,
\end{aligned}
$$

where $n_{i}$ is the number of input layer nodes, $n_{o}$ is the number of output layer nodes, $k[1,10]$. According to the empirical formula (2), the range of the number of neurons in the hidden layer is estimated to be $[5,14]$, then space is slightly expanded to $[3,16]$ during the training, and the BP neural network is used to train repeatedly.

To sum up, the number of neurons in the input layer, hidden layer, and output layer is 14,15 , and 1 , the activation function of neurons is the sigmoid function, the error function (objective function) is a quadratic mean square function, Gaussian random variable with a mean value of 0 and standard deviation of nin is selected as initialization weight, and Gaussian random variable with the mean value of 0 and standard deviation of 1 is selected as initialization threshold.

$$
\gamma_{j}=f^{\prime}\left(\text { net }_{j}\right) \sum_{k} \delta v_{j k}=l_{j}\left(1-l_{j}\right) \sum_{k} \delta v_{j k}
$$

where $y j$ is the target value of the $\mathrm{K}$ nodes and $z k$ is the output value of the K nodes. After the salary forecast model is established, the training can be started. The training flowchart of the model is represented in Figure 3.

In the previous introduction, BP neural network adopts the gradient descent method in the reverse propagation update. According to the amount of data used in each update, the gradient descent method can be divided into three forms: batch gradient descent method, random gradient descent method, and small-batch gradient descent method. Each update is called batch gradient descent which uses all samples to update. Because batch gradient descent needs to use all training samples at every iteration, when the number of training datasets is large, the time of one iteration update will be longer, the cumulative error of all samples will decrease down to a certain extent, and eventually, the training process will be slow. As a result, the batch gradient descent training process may fall into local optimal but cannot achieve global optimization. The method of updating only a single sample is called stochastic gradient descent (SGD). Because SGD only uses a single sample for each iteration update and causes parameter updates very frequently, the update between different sample data may offset each other, which makes each update not advance toward the overall optimization direction, and it is prone to oscillation phenomenon, but this oscillation also makes it possible to jump out of local optimal position and reach global optimal position. The minibatch gradient descent (MBGD) is a compromise method to solve the problems existing in BGD and SGD. It divides the whole sample dataset into small sample datasets and takes the cumulative error gradient of each small sample dataset as the estimation value of the real gradient of the whole sample. When the sample size is large enough, the local error gradient value is approximately equal to the total sample error gradient value. The small-batch gradient descent method can have both bad and SGD advantages.

\section{Experimental Simulations and Result Analysis}

This section of the paper represents the experimental results carried out via different experiments and their analysis. All the experiments were performed on a laptop computer system having the following specifications: Intel Core-i5, 7th generation, processor of $2.4 \mathrm{GHz}, 16 \mathrm{~GB}$ of RAM, and the operating system installed on the system being Microsoft Windows 10). Anaconda Jupyter Notebook is used as an IDE for carrying out the simulations. Python has been used as a language for the implementation and for generating the simulation results. The list of python packages used in this study was Keras, TensorFlow, seaborn, Numpy, Matplotlib, and Sklearn, and so on.

4.1. Results of Salary Forecast Model Based on BP Neural Network. This subsection demonstrates the simulation results carried out via the salary forecast model based on BP neural network. All the simulation results were attained using a different number of neurons and iterations as shown in Tables 1 and 2. Table 1 shows the training and validation accuracies of the salary forecast model based on the BP neural network, using the data size of 1000 samples. The dataset samples were divided into training and testing samples, the size of the training dataset was 700, the size of the testing dataset was 300, the number of neurons in each hidden layer was trained 30 times, and finally, the average value of the 30 times results was calculated. The execution process reached its termination when the number of iterations reaches 250 .

From Table 1, it is quite obvious that the training and validation accuracies increase as the number of neurons in the hidden layer is increased. Figure 4 shows the training and validation accuracies of the salary forecast model based on the BP neural network.

Figure 4 illustrates that the training and validation accuracies increase with the increase in the number of iterations. The highest training accuracy of 89.98 was attained at the 250th iteration. The highest validation accuracy of 84.05 was also conquered at the 250th iteration.

Table 2 shows the training and validation loss of the salary forecast model based on the BP neural network. The training and validation loss of the BP neural network decrease as the number of neurons in the hidden layer is increased. 


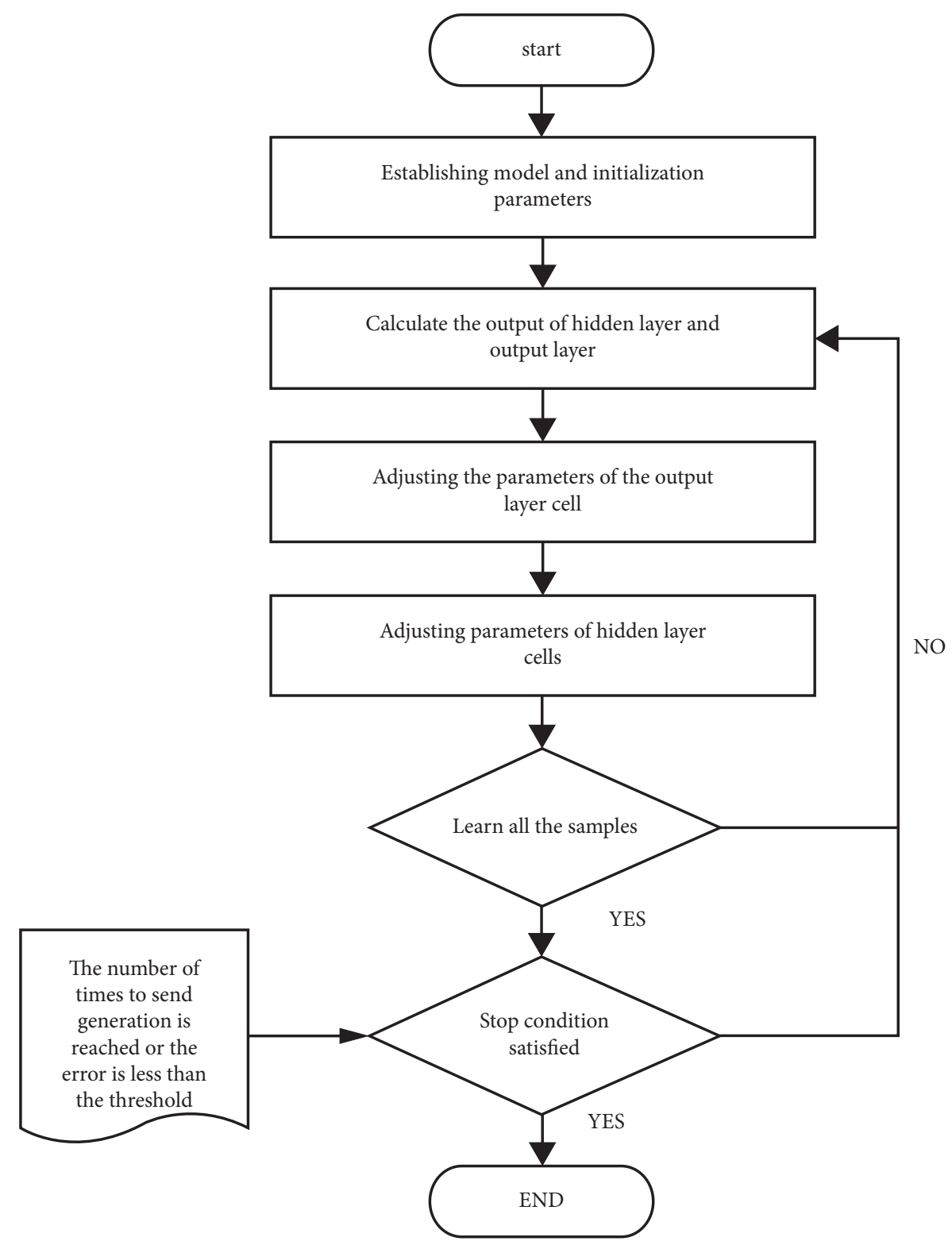

FIgURE 3: Training flowchart of the wage prediction model.

TABle 1: Training and testing accuracies of BP neural network using a different number of neurons in the hidden layer.

\begin{tabular}{lcc}
\hline Number of neurons & Training accuracy & Validation accuracy \\
\hline 3 & 86.66 & 80.04 \\
4 & 87.04 & 80.20 \\
5 & 87.30 & 80.44 \\
6 & 87.44 & 80.52 \\
7 & 87.46 & 80.72 \\
8 & 87.80 & 80.96 \\
9 & 87.94 & 81.22 \\
10 & 88.00 & 81.44 \\
11 & 88.52 & 81.73 \\
12 & 88.64 & 81.93 \\
13 & 89.00 & 82.17 \\
14 & 89.50 & 83.08 \\
15 & 89.80 & 83.86 \\
16 & 89.98 & 84.05 \\
\hline
\end{tabular}

We also performed multiple experiments using different gradient descent techniques, that is, batch gradient descent (BGD), stochastic gradient descent (SGD), and minibatch gradient descent (MBGD). To show the training effect of BGD, SGD, and MBGD in an effective way, the loss function curve of these techniques during training is shown in Figure 5. Among them, the horizontal coordinate in Figure 5 is the iteration update times, the ordinate is the loss function (cost), the training sample size is 200, and the small-batch sample size is set to 20. So, BGD performed 200 cycles (read 200 rounds of dataset), MBGD performed 20 cycles, and SGD performed a single cycle.

From Figure 6, it can be seen that when the iteration update time is the same, the convergence results of the three gradient descent methods are relatively close, but the loss change curve is very different: SGD curve has a large fluctuation, BGD curve is relatively smooth, and MBGD is 
TABLE 2: Training and validation loss of BP neural network using a different number of neurons in the hidden layer.

\begin{tabular}{lcc}
\hline Number of neurons & Training loss & Validation loss \\
\hline 3 & 0.009975 & 0.010725 \\
4 & 0.009958 & 0.010724 \\
5 & 0.009950 & 0.010723 \\
6 & 0.009954 & 0.010720 \\
7 & 0.009947 & 0.010716 \\
8 & 0.009944 & 0.010710 \\
9 & 0.009940 & 0.010690 \\
10 & 0.009938 & 0.010660 \\
11 & 0.009932 & 0.010630 \\
12 & 0.009928 & 0.010595 \\
13 & 0.009926 & 0.010580 \\
14 & 0.009920 & 0.010553 \\
15 & 0.009916 & 0.010510 \\
16 & 0.009912 & 0.010502 \\
\hline
\end{tabular}

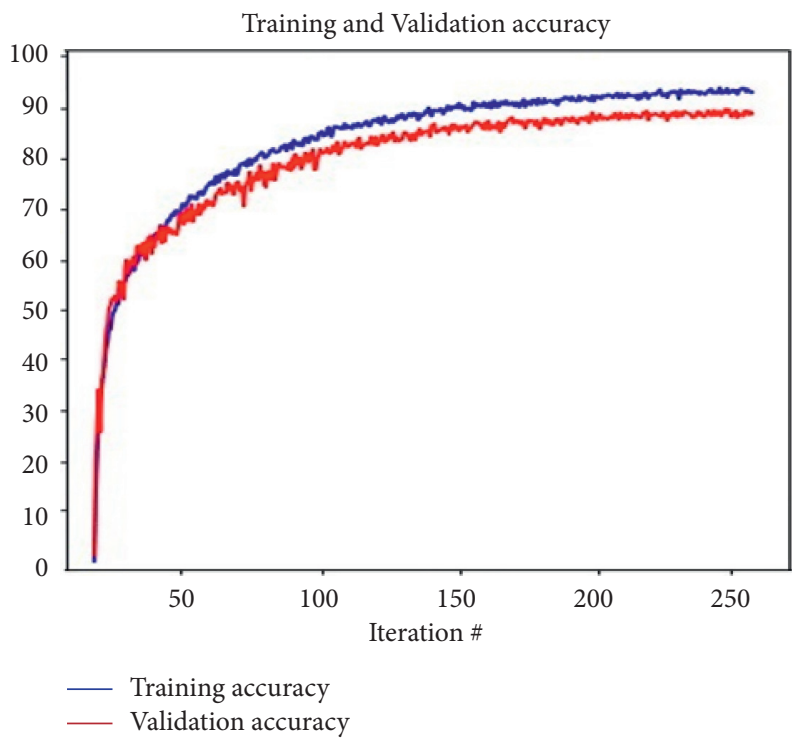

FIGURE 4: Training and validation accuracies of BP neural network.

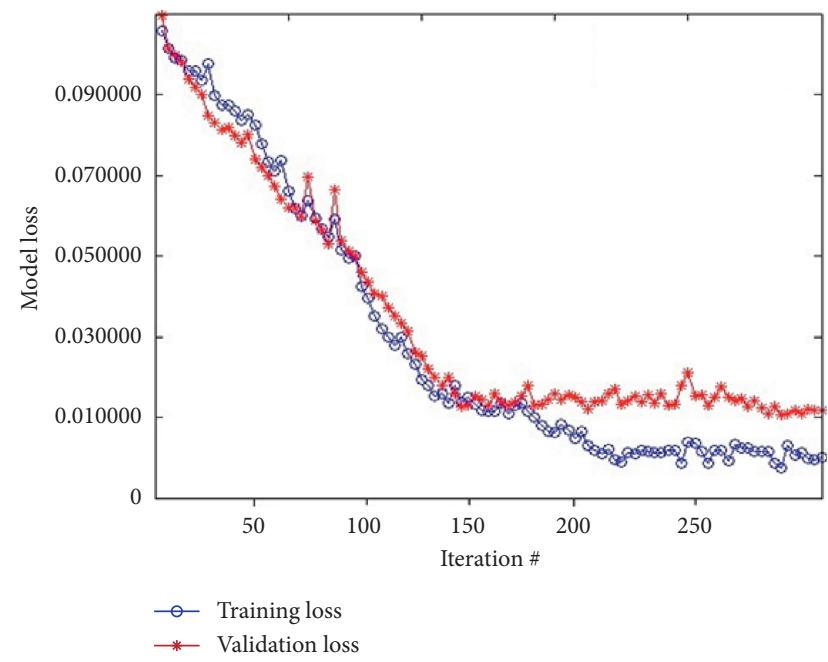

Figure 5: Training and validation loss of BP neural network.

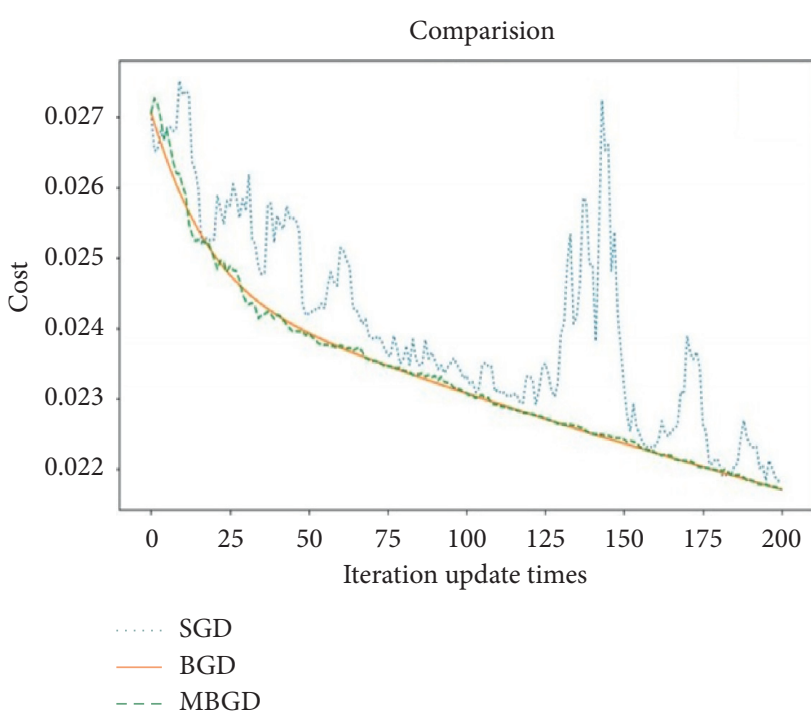

FIGURE 6: Comparison of SGD, BGD, and MBGD iterative update process.

between SGD and BGD and is relatively stable. The training speed of SGD, BGD, and MBGD is different, and the training period of SGD, BGD, and MBGD is different, which is different from that of SGD, BGD, and MBGD. If the sample space is large, the training speed of the investigated three techniques will be more obvious, especially SGD and BGD. In short, the training speed of SGD is fast and iteration update is frequent, but there is a fluctuation that easily produces oscillation. BGD is stable and iteration update is less, but the training speed is slow. MBGD is a compromise scheme, which has a fast training speed and is relatively stable.

The three-layer BP neural network model used for the salary prediction consists of a different number of neurons in different layers. The number of neurons in the input layer, hidden layer, and output layer is 14,15 , and 1, the activation function is the sigmoid function, the error function is quadratic mean square error (QMSE), and the network parameters are updated by using small-batch gradient descent method (SBGD) and mixed optimization method Nadam. The method used in this experiment divides the dataset into two parts, that is, training and testing. The total sample space selected for the experiment was 1000, of which 900 samples were selected for training while the rest of the 100 samples were selected for testing and validation purpose.

Figure 7 shows the adaptation process and comparison of training effect of various optimization algorithms. In Figure 7, the ordinate is the loss function (cost) and the abscissa is the period (iteration). As can be seen from Figure 7, Adam and Nadam have better performance than other optimization algorithms in terms of convergence speed, whereas Nadam is slightly better than Adam in terms of overall performance.

To better reflect the performance of Nadam based salary prediction model, the comparison results between Nadam and other ML regression algorithms are given here. The size of the training sample space is 900 , and the size of the test 


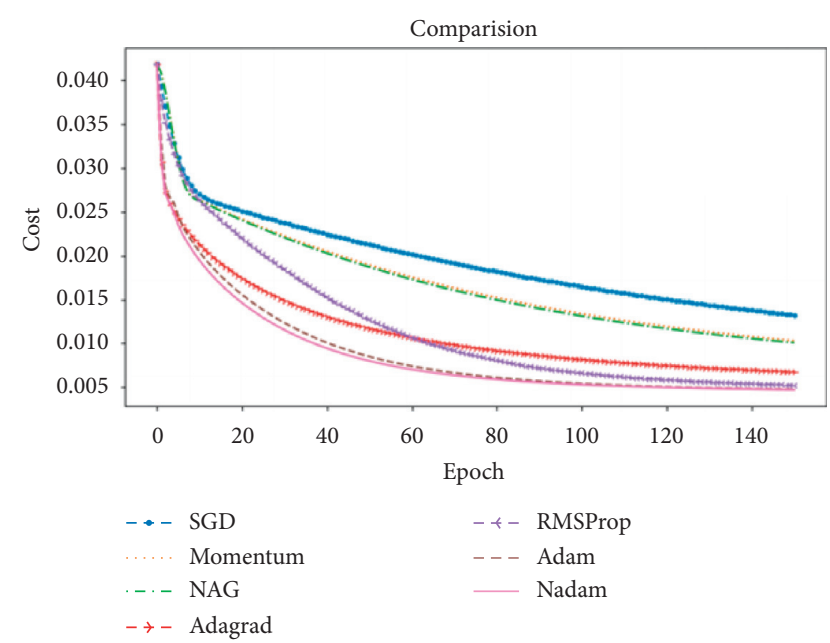

Figure 7: Comparison of convergence rates of various optimization methods.

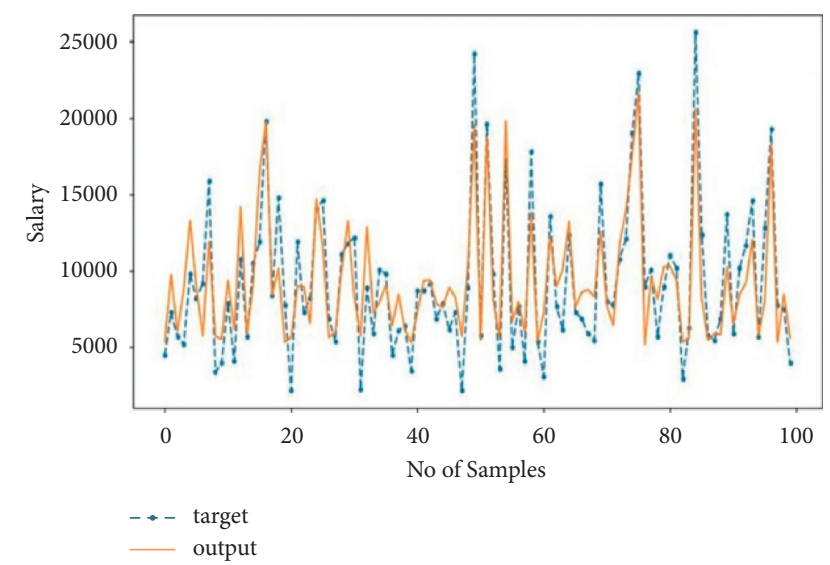

Figure 8: Fitting effect of Nadam based salary forecasting model.

sample space is 100. Each algorithm is tested 10 times, and the best test score is obtained. It can be observed that the results of the Nadam optimized salary prediction model in the test set is relatively higher as compared to the other utilized algorithms. Finally, the fitting effect of the Nadam salary prediction model is calculated and is shown in Figure 8.

It can be seen from Figure 8 that the prediction fitting effect of the Nadam based salary prediction model is comparatively higher as compared to the rest of the techniques. Although the normalization and antinormalization of salary data in model training may lead to some errors in calculation, this error is still within the controllable range and has no great influence on the final results of the model. Further, the salary prediction model optimized by Nadam has a fast convergence speed and good prediction effect and has good accuracy as well, so it has certain practical and reference value.

\section{Conclusion}

Globalization and worldwide economic integration have had a considerable impact on China's economic development, including the business community, in past few years. This results in disparities in many facets of business operations and trade, such as business thinking, observations, confidence, client psychology, behavioral culture, and salary prediction. As a result, for sustainable and adaptive development in the new context, each small- and medium-sized firm must effectively leverage supportive solutions from the government, social media platforms, and small- and medium-sized enterprises themselves. This study mainly focuses on the application and implementation of the BP neural network-based salary forecasting model. Multiple experiments have been performed by using a different number of neurons in the hidden layers and a different number of iterations in order to check the stability and effectiveness of the proposed model. This study also uses the gradient descent algorithm and its types by performing multiple experiments for each of its types. Among the implemented types of gradient descent algorithm, Nadam showed good performance as compared to its other types. BP neural network-based salary forecasting model performed excellently by attaining the training accuracy of $89.98 \%$ and validation accuracy of $84.05 \%$. The proposed BP neural network-based salary forecasting model is anticipated to be of great help in human resource management systems.

\section{Data Availability}

The data used to support the findings of this study are included within the article.

\section{Conflicts of Interest}

The author declares that there are no conflicts of interest regarding the publication of this study.

\section{References}

[1] Y. Lu, Research on the Application of Data Mining Technology in Human Resource Management Systems, Beijing University of Posts and telecommunications, Beijing, China, 2020.

[2] L. Y. Wang and C. I. Peng, "Application of decision tree algorithm in human resource recommendation technology," Modern electronic technology, vol. 44, no. 3, pp. 105-110, 2021.

[3] T. Liu, M. Liu, H. Lihong, W. Luo, and W. Zheng, "Exploration of human resource evaluation model based on ERP," Energy Technology, vol. 18, no. 9, pp. 90-93, 2020.

[4] Q. Xia and F. Huang, "Data-driven human resource management: review and prospect," China personnel science, vol. 12, no. 6, pp. 72-87, 2020.

[5] J. Tang, Programmer Recommendation System Based on GitHub, Yangzhou University, Yangzhou, China, 2020.

[6] Y. Li, S. Li, Y. song, F. Zhang, and X. Zhou, "Human Resource Recommendation Based on K-means clustering algorithm of spark platform," Journal of Jinan University (Natural Science \& Medicine Edition), vol. 34, no. 5, pp. 430-435, 2020.

[7] L. Qi, Design and Implementation of a Personalized Recommendation System for enterprise Training Resources, Harbin Institute of Technology, Harbin, China, 2018.

[8] G. Liang, Human Resource Recommendation Algorithm Based on Lifting Tree and Neural Network, South China University of Technology, Guangzhou, China, 2018. 
[9] C. Lin, Human Resource Recommendation Algorithm Based on Hybrid Genetic Ensemble Learning, South China University of Technology, Guangzhou, China, 2018.

[10] Z. Gu, Human Resource Recommendation Algorithm Based on the Hidden Semantic Model and Deep forest, South China University of Technology, Guangzhou, China, 2018.

[11] S.. Research, "On job recommendation of AI companies based on data mining," Value Engineering, vol. 36, no. 34, pp. 42-44, 2017.

[12] Z. Li, Human Resource Hybrid Recommendation Algorithm Based on Spark, South China University of Technology, Guangzhou, China, 2017.

[13] W. Chen, Research on Human Resource Recommendation Algorithm Based on Deep Learning, South China University of Technology, Guangzhou, China, 2017.

[14] F. Meng, Application Research of Human Resource Recommendation Engine Based on Hybrid Recommendation Algorithm, Beijing University of Technology, Beijing, China, 2014.

[15] P. N. Q. Huong, "The quality OF human resources IN small and medium enterprises: current situation, reason, recommendations ON solutions," Journal of Natural Remedies, vol. 21, pp. 73-81, 2021.

[16] D. L. Stone and J. H. Dulebohn, Emerging Issues in Theory and Research on Electronic Human Resource Management (eHRM), Elsevier, Amsterdam, Netherlands, 2013.

[17] J. Heikkila, "A delphi study on e-HRM: future Directions," in Proceedings of the 3rd European Academic Workshop on Electronic Human Resource Management, pp. 229-249, Bamberg, German, May 2010.

[18] M. Berg, The Age of Manufactures, 1700-1820: Industry, Innovation and Work in Britain, Routledge, Oxford, England, 2005.

[19] C. Trebilcock, The Industrialisation of the Continental Powers 1780-1914, Routledge, Oxford, England, 2014.

[20] F. W. Taylor, Scientific Management, Routledge, Oxford, England, 2004.

[21] O. Yabanci, "From human resource management to intelligent human resource management: a conceptual perspective," Human-Intelligent Systems Integration, vol. 1, no. 2-4, pp. 101-109, 2019.

[22] A. Iqbal, "The strategic human resource management approaches and organisational performance: the mediating role of creative climate," Journal of Advances in Management Research, vol. 8, 2019.

[23] R. Mathis and J. Jackson, Human Resource Management Mason, Thomson South-Western, Mason, OH, USA, 2008.

[24] M. Armstrong and S. Taylor, Armstrong's Handbook of Human Resource Management Practice, Kogan Page, London, England, 2009.

[25] H. Francis and A. Keegan, "The changing face of HRM: in search of balance," Human Resource Management Journal, vol. 16, no. 3, pp. 231-249, 2006.

[26] S. Strohmeier, "Research in e-HRM: review and implications," Human Resource Management Review, vol. 17, no. 1, pp. 19-37, 2007.

[27] J. H. Marler and E. Parry, "Human resource management, strategic involvement and e-HRM technology," International Journal of Human Resource Management, vol. 27, no. 19, pp. 2233-2253, 2016.

[28] L. Ma and M. Ye, "The role of electronic human resource management in contemporary human resource management," Open Journal of Social Sciences, vol. 03, no. 04, pp. 71-78, 2015.
[29] S. Panos and V. Bellou, "Maximizing E-HRM outcomes: a moderated mediation path," Management Decision, vol. 54, 2016.

[30] M. Thite, e-HRM: Digital Approaches, Directions \& Applications, Routledge, Oxford, England, 2018.

[31] Y. Pan, "Heading toward artificial intelligence 2.0," Engineering, vol. 2, no. 4, pp. 409-413, 2016.

[32] H. Shah and K. Warwick, "Testing Turing's five minutes, parallel-paired imitation game," Kybernetes, vol. 39, 2010.

[33] Y. Shiroishi, K. Uchiyama, and N. Suzuki, "Society 5.0: for human security and well-being," Computer, vol. 51, no. 7, pp. 91-95, 2018.

[34] S. I. Team, "Realization OF society 5.0 BY utilizing precision agriculture into smart agriculture IN naro, Japan," in International Workshop on ICTs for Precision Agriculture, p. 58, Wuhan, China, 2019.

[35] P. Skobelev and S. Y. Borovik, "On the way from industry 4.0 to industry 5.0: from digital manufacturing to digital society," Industry, vol. 2pp. 307-311, Suceava, Romania, 2017. 